\title{
Fratura de Stent como Causa de Reestenose em Stent Farmacológico
}

\author{
Júlio César Machado Andréa1, José Ary Boechat e Salles, \\ Filipe Goldberg ${ }^{1}$, Leandro Cortes ${ }^{1}$, Hélio Roque Figueira ${ }^{1}$
}

\section{RESUMO}

Os stents coronários são usados em mais de $90 \%$ das intervenções coronárias percutâneas. Entretanto, sua eficácia está limitada pela ocorrência de reestenose, variando de $15 \%$ a $50 \%$ dos casos, dependendo da morfologia da lesão e da presença de co-morbidades (como, por exemplo, diabetes melito e insuficiência renal). Nos últimos anos, os stents farmacológicos provaram ser eficazes em suprimir a hiperplasia neo-intimal, reduzindo a taxa de reestenose para um dígito. Neste artigo, os autores descrevem um caso de reestenose em stent com eluição de sirolimus relacionada à fratura do stent.

DESCRITORES: Contenedores, efeitos adversos. Reestenose coronária. Sirolimus. Falha de equipamento.
$\mathrm{N}$ os últimos anos, os stents farmacológicos (SF) provaram ser eficazes em suprimir a hiperplasia neo-intimal, reduzindo a reestenose intra-stent entre $60 \%$ e $75 \%$, dependente da morfologia da lesão e da associação de co-morbidades (como, por exemplo, diabetes melito e insuficiência renal).

A reestenose dos SF relaciona-se à injúria nas bordas provocada pelo balão (pré e pós-dilatação), à não cobertura total da lesão abordada (geographic miss), à assimetria das hastes, à ruptura do polímero, à resistência e/ou à fratura do stent. Descrevemos um caso de reestenose em stent com eluição de sirolimus (SES) relacionada à fratura do stent.

\section{RELATO DO CASO}

Homem de 61 anos, com hipertensão arterial e dislipidemia, em angina estável classe funcional II-III, de acordo com a Canadian Cardiovascular Society (CCS). Em março de 1999, o paciente foi submetido

\footnotetext{
1 Hospital CardioTrauma Ipanema - Rio de Janeiro, RJ. Correspondência: Júlio Andréa. Av. Sernambetiba, 3300 - BI. 2/1301

- Barra da Tijuca - Rio de Janeiro, RJ - CEP 22630-010

E-mail: julioandrea@cardiol.br

Recebido em: 22/5/2007 • Aceito em: 27/11/2007
}

\section{SUMMARY}

\section{Stent Fracture and Restenosis of Drug-Eluting-Stents}

The latest great revolution in the management of restenosis has been the introduction of the drug-eluting-stents (DES). They have been proven very effective in suppressing neointimal proliferation and reduces restenosis rates to single digit numbers. A case of DES strut fracture-induced restenosis is described.

DESCRIPTORS: Stents, adverse effects. Coronary restenosis. Sirolimus. Equipment failure.

a cirurgia de revascularização miocárdica (enxertos de artéria torácica interna esquerda-descendente anterior e venosos para primeira diagonal e primeira marginal esquerda).

Em março de 2006, o paciente apresentou episódio de angina instável progressiva e foi submetido a angiografia coronária, que demonstrou enxertos arteriais pérvios e venosos ocluídos, oclusão da artéria descendente anterior, artéria coronária direita pérvia, estenose segmentar suboclusiva da artéria circunflexa e função ventricular esquerda preservada. Optou-se por reconstrução endoluminal da artéria circunflexa, com implante de dois SES 3,0 x $18 \mathrm{~mm}$ e 2,5 x $33 \mathrm{~mm}$ em overlapping e impactação com balão 3,0 × $20 \mathrm{~mm}$ a $14 \mathrm{~atm}$, com excelente resultado angiográfico (Figura 1).

Após nove meses, o paciente desenvolveu quadro de angina aos esforços. O estudo funcional com cintilografia miocárdica demonstrou isquemia de parede lateral. A angiografia revelou reestenose focal de $90 \%$ na porção distal do stent $2,5 \times 33 \mathrm{~mm}$, exatamente no local da anastomose distal do enxerto venoso aortaprimeira marginal esquerda, com movimento de torção do vaso nesse ponto (Figura 2). A análise com ultrasom intracoronário (USIC) demonstrou marcada hiperplasia neo-intimal, hipoexpansão, ausência das hastes 
do stent correspondendo à fratura do stent e ateroma distal ao stent (Figura 3).

Com base nesses achados ultra-sonográficos, a reestenose foi tratada com implante primário de stent com eluição de paclitaxel (PES) (Taxus $\left.{ }^{\circledR}\right) 3,0 \times 20$ mm, impactado a 16 atm, com excelente resultado angiográfico e ultra-sonográfico (Figura 4).

\section{DISCUSSÃO}

O uso de SES e/ou de PES reduziu, acentuadamente, as taxas de reestenose, quando comparados aos stents convencionais $(\mathrm{SC})^{1,2}$.

A reestenose angiográfica dos SF está abaixo dos $10 \%$ e habitualmente está relacionada à injúria nas

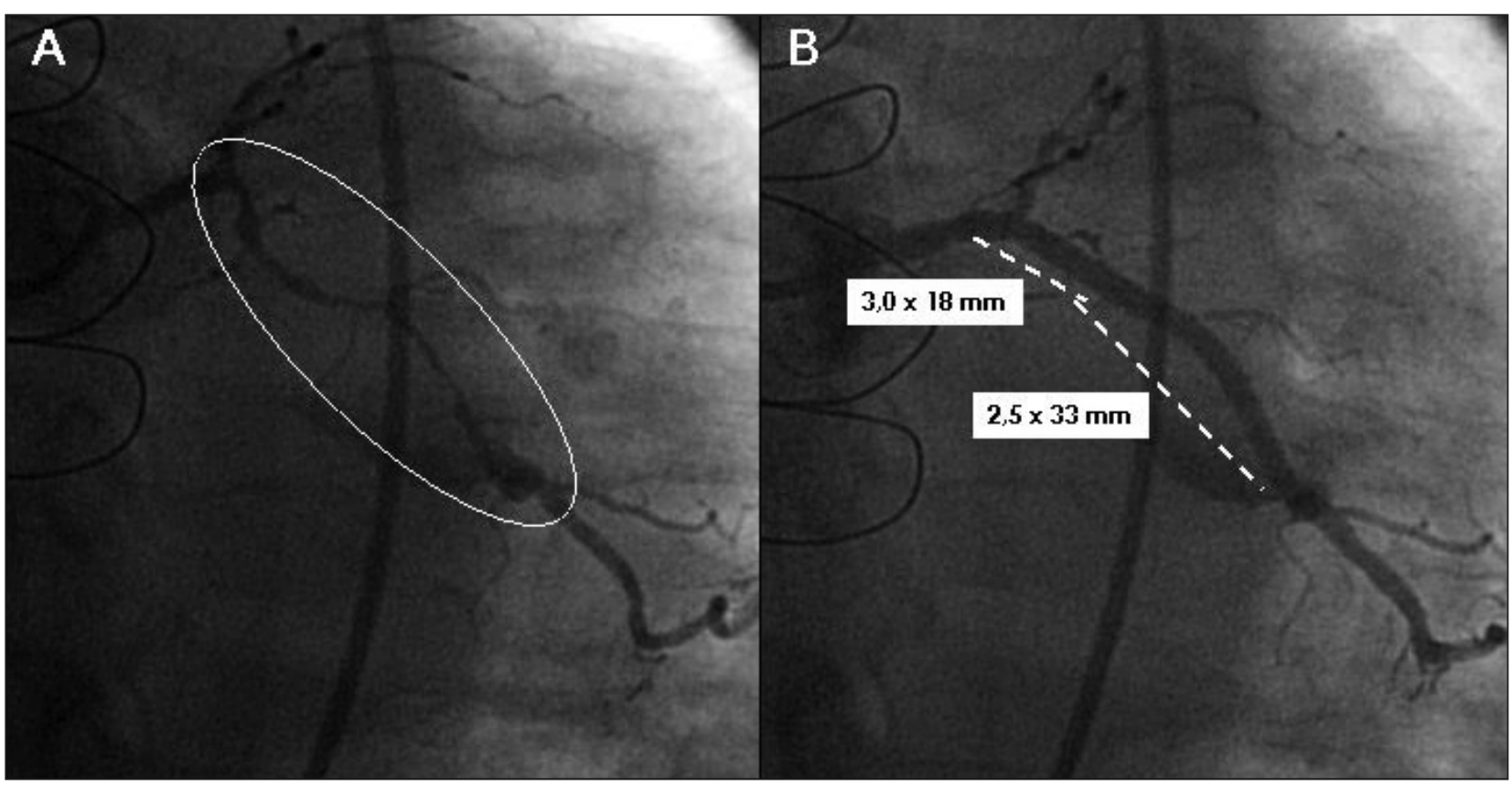

Figura 1 - Angiografia antes (A) e após (B) implante de dois stents com eluição de sirolimus em overlapping.

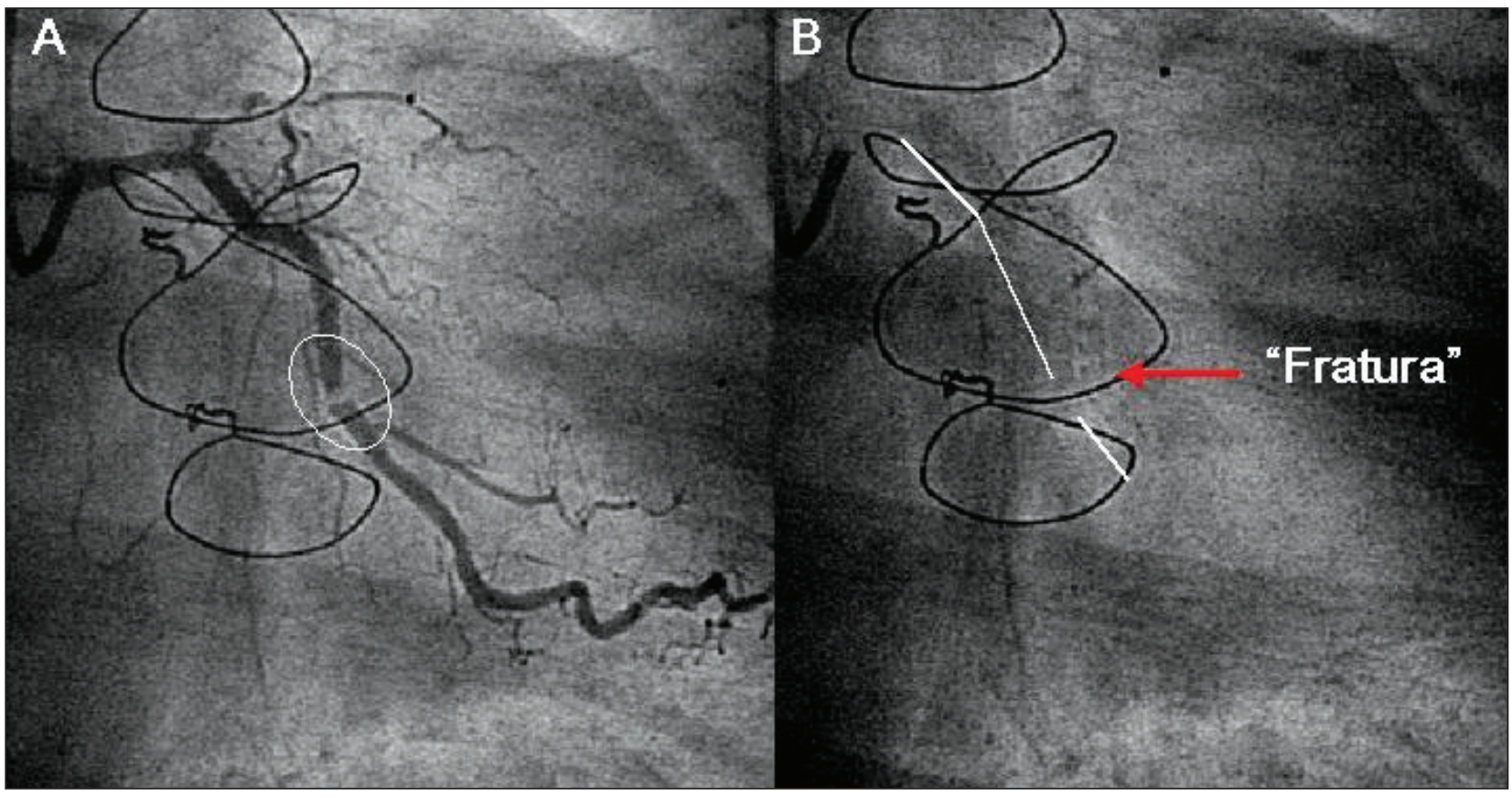

Figura 2 - Angiografia demonstrando reestenose focal (A) e aspecto da "fratura" do stent (B). 

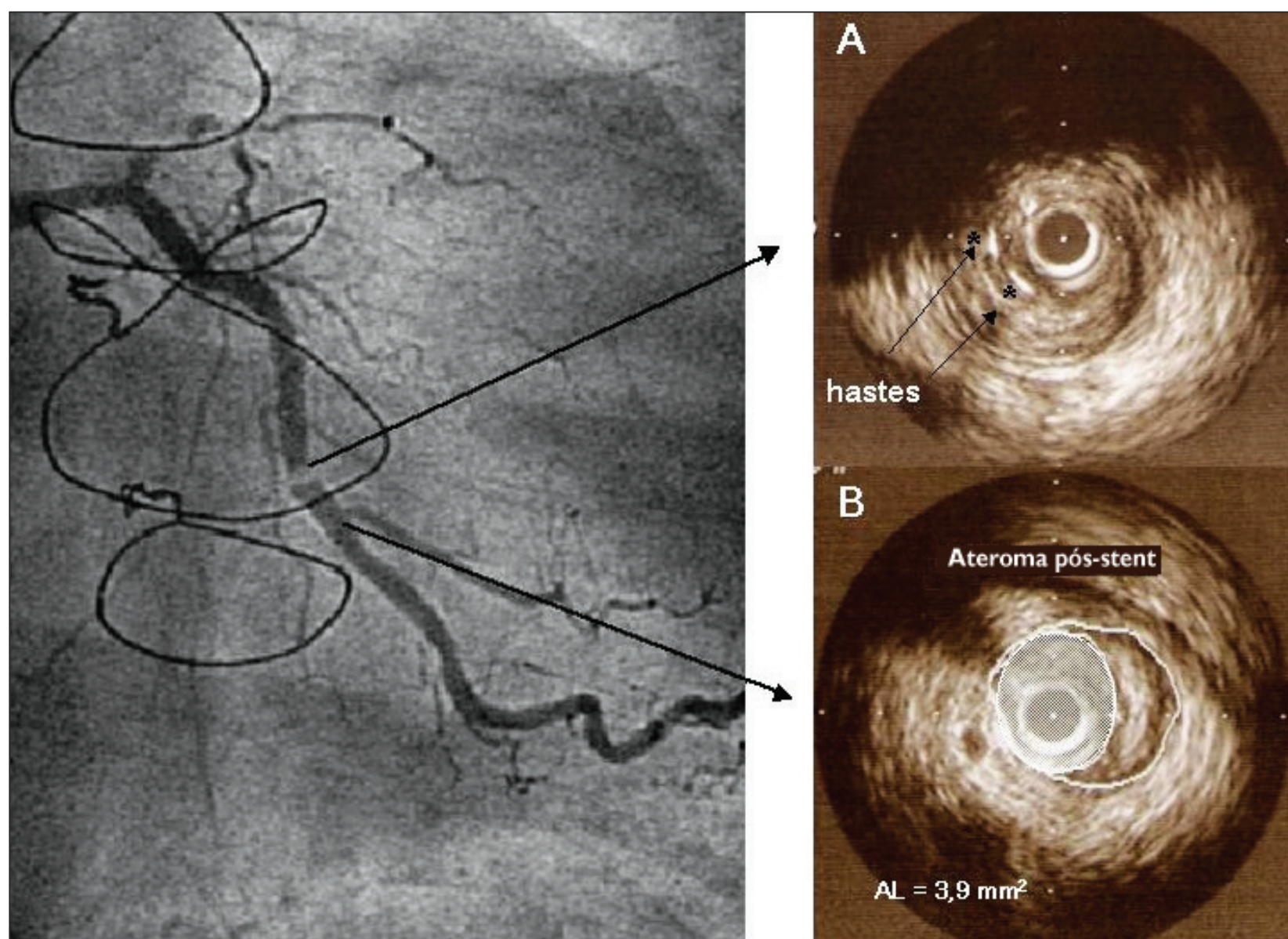

$\mathrm{AL}=3,9 \mathrm{~mm}^{2}$

Figura 3 - Ultra-sonografia intracoronária (USIC). Em A, marcada hiperplasia neo-intimal e hastes "soltas"; em B, ateroma pós-stent.

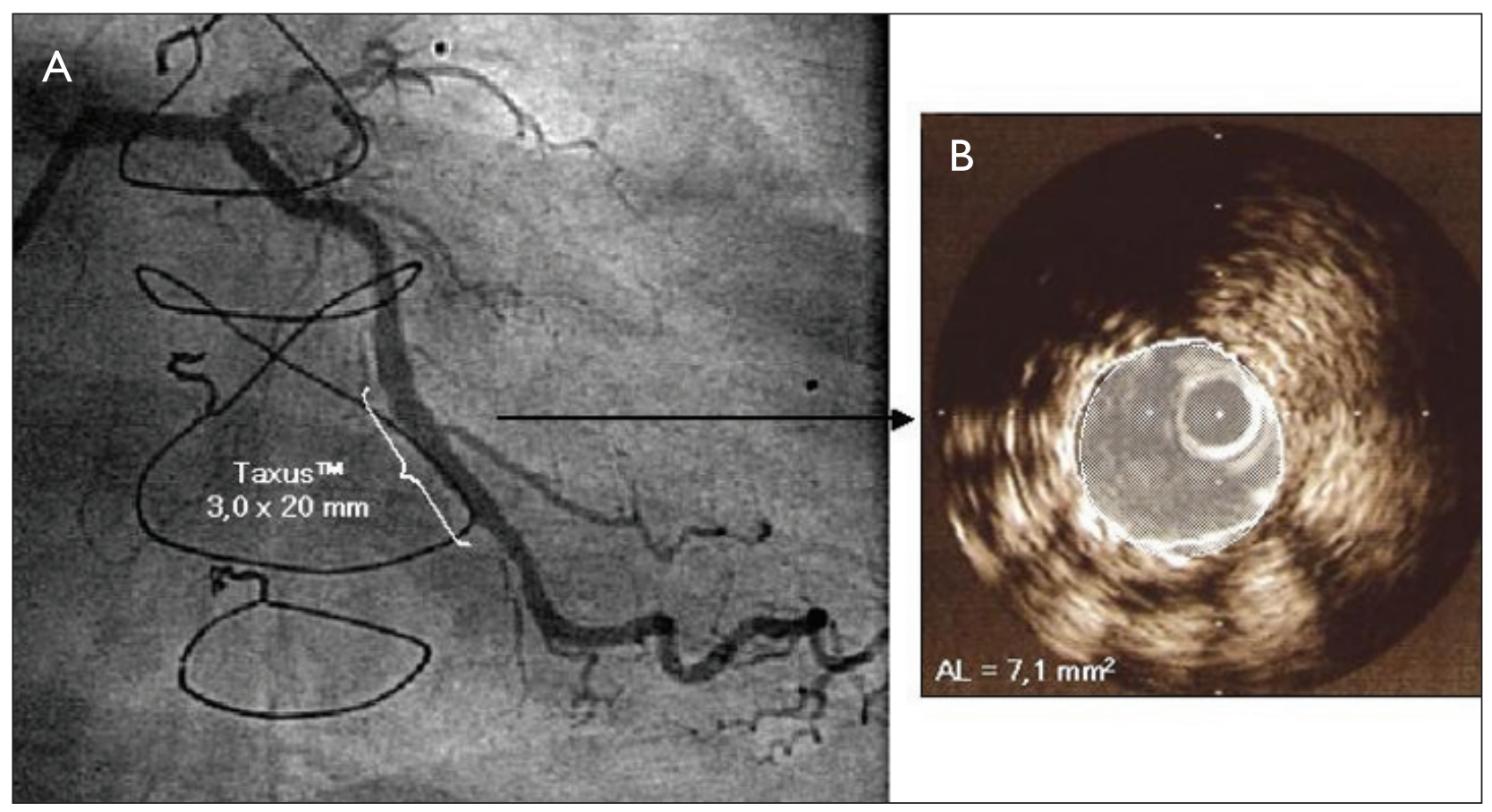

Figura 4 - Angiografia (A) e ultra-sonografia intracoronária (B) pós-implante de stent com eluição de paclitaxel. 
bordas provocada pelo balão (pré e pós-dilatação), à não cobertura total da lesão abordada (geographic miss) ${ }^{3}$, à assimetria das hastes, à hipoexpansão, à ruptura do polímero, à resistência à droga, à hipersensibilidade à droga e/ou polímero e à fratura do stent ${ }^{4,5}$.

A fratura de stent, definida no USIC como diminuição do número de hastes durante o seguimento em relação ao momento do implante, favoreceria a distribuição não-uniforme da droga, podendo contribuir para o desenvolvimento da reestenose ${ }^{6}$. No presente caso, não dispúnhamos da avaliação ultra-sonográfica do implante do SES; entretanto, no seguimento, observamos nítida redução das hastes associada à hipoexpansão da endoprótese, que podem ter contribuído para hiperplasia neo-intimal localizada.

A fratura é relativamente incomum, sendo o mecanismo potencial a fadiga mecânica do material dos stents implantados em regiões de grande curvatura ${ }^{7}$, onde o ciclo cardíaco exerce grande estresse, tornandoos mais vulneráveis, especialmente os mais longos.

Em conclusão, a reestenose do SF decorrente de fratura do stent envolve a estimulação mecânica local pelas hastes "quebradas" da parede do vaso, resultando em inflamação e conseqüente hiperplasia neo-intimal. Além disso, a fratura acarreta a destruição da arquitetura do stent, ocasionando a distribuição não-uniforme da droga $a^{8-10}$, o que contribui para reestenose intensamente localizada, como a demonstrada no caso relatado. Estudos com USIC ajudarão a quantificar a importância da fratura do stent nesse cenário ${ }^{3}$.

\section{REFERÊNCIAS BIBLIOGRÁFICAS}

1. Moses JW, Leon MB, Popma JJ, Fitzgerald PJ, Holmes DR, O'Shaughnessy C, et al. Sirolimus-eluting stents versus standard stents in patients with stenosis in a native coronary artery. N Engl J Med. 2003;349(14):1315-23.

2. Stone GW, Ellis SG, Cox DA, Hermiller J, O'Shaughnessy C, Mann JT, et al. A polymer-based, paclitaxel-eluting stent in patients with coronary artery disease. N Engl J Med. 2004;350(3):221-31.

3. Brilakis ES, Maniu C, Wahl M, Barsness G. Unstable angina due to stent fracture. J Invasive Cardiol. 2004;16(9):545.

4. Min PK, Yoon YW, Moon Kwon H. Delayed strut fracture of sirolimus-eluting stent: a significant problem or an occasional observation? Int J Cardiol. 2006;106(3):404-6.

5. Sianos G, Hofma S, Ligthart JM, Saia F, Hoye A, Lemos PA, et al. Stent fracture and restenosis in the drug-eluting stent era. Catheter Cardiovasc Interv. 2004;61(1):111-6.

6. Takebayashi H, Mintz GS, Carlier SG, Kobayashi Y, Fujii K, Yasuda T, et al. Nonuniform strut distribution correlates with more neointimal hyperplasia after sirolimus-eluting stent implantation. Circulation. 2004;110(22):3430-4.

7. Murphy BP, Savage P, McHugh PE, Quinn DF. The stressstrain behavior of coronary stent struts is size dependent. Ann Biomed Eng. 2003;31(6):686-91.

8. Chowdhury PS, Ramos RG. Images in clinical medicine. Coronary-stent fracture. N Engl J Med. 2002;347(8):581.

9. Halkin A, Carlier S, Leon MB. Late incomplete lesion coverage following Cypher stent deployment for diffuse right coronary artery stenosis. Heart. 2004;90(8):e45.

10. Hamilos MI, Papafaklis MI, Ligthart JM, Serruys PW, Sianos G. Stent fracture and restenosis of a paclitaxel-eluting stent. Hellenic J Cardiol. 2005;46(6):439-42. 